\title{
Public Sector Corruption and Trust in the Private Sector
}

\author{
Robert Gillanders \\ Hanken School of Economics and HECER \\ and \\ Olga Neselevska \\ Hanken School of Economics and HECER
}

Discussion Paper No. 403

April 2016

ISSN 1795-0562

HECER - Helsinki Center of Economic Research, P.O. Box 17 (Arkadiankatu 7), FI-00014 University of Helsinki, FINLAND, Tel +358-2941-28780, E-mail info-hecer@helsinki.fi, Internet www.hecer.fi 


\title{
Public Sector Corruption and Trust in the Private Sector *
}

\begin{abstract}
In this paper we use data from the Afrobarometer surveys to demonstrate that there is an undesirable spill-over from petty corruption in the public sector to trust in private sector institutions. Our results show that experiencing bribery in the course of one's interactions with the public sector lowers one's trust in big private corporations, small businesses, and local traders. This finding holds even when we allow for perceptions of political corruption to enter the specification. We do not find any significant association between a measure of interpersonal trust and bribery experience which suggests that our findings with regards to market institutions are not driven by corruption lowering trust in general. Having to pay a bribe for household services, which is perhaps the setting most like a private sector transaction, is the corrupt interaction most strongly associated with the decline in private sector trust. We find some evidence that the spill-over is larger in democracies than in non-democratic regimes. Given the importance of trust in market institutions for the efficient functioning of an economy, our findings thus point to a previously unknown and potentially substantial cost of corruption and add to the case for anti-corruption efforts.
\end{abstract}

JEL Classification: D73, K4, O10, O55

Keywords: bribery, corruption, corruption experiences, corruption perceptions, private sector trust, sub-Saharan Africa, trust

Robert Gillanders

Hanken School of Economics

Department of Economics

P.O. Box 479

FI-00101 Helsinki

FINLAND

e-mail: rgillanders@gmail.com
Olga Neselevska

Hanken School of Economics

Department of Economics

P.O. Box 479

FI-00101 Helsinki

FINLAND

e-mail: o.neselevska@gmail.com

* We are grateful to Laura Arranz Aperte, Tom Berglund, Michael Breen, Amadou Boly, Mika Haapanen, Ilpo Kauppinen, Topi Miettinen, Staffan Ringbom, Smriti Sharma, Saurabh Singhal, Rune Stenbacka, and seminar participants at the Finnish Economics Association 2016 annual conference, UNU-WIDER, HECER, and the Hanken Centre for Corporate Governance for helpful comments and suggestions. Neselevska gratefully acknowledges financial support from the Kone foundation. 


\section{INTRODUCTION}

There is a long standing literature that argues that trust is an essential economic lubricant. For example, trust has been shown to be important for growth and development (Knack and Keefer, 1997; Zak and Knack, 2001). It is most often asserted that this operates via a reduction in transaction costs. On the other hand, corruption, the abuse of public power for private gain, has been found by many researchers to be sand in the wheels of an economy. Macro evidence suggests that corruption is detrimental to economic growth (Mauro, 1995), though perhaps only in institutional settings that are otherwise strong (Aidt et al, 2008), and to foreign direct investment inflows (Wei, 2000). Similarly, Fisman and Svensson (2007) find that bribery has a negative effect on firm growth. This paper examines the relationship between these two important social forces in that we ask if individuals who experience corruption in their dealings with the public sector are more or less likely to trust the private sector.

It is not obvious what the association between experiencing public sector corruption and trust in the private sector should be. On the one hand, if one has to pay a bribe to an agent of one's state, one may feel more inclined to view favourably the institutions of the private sector. That is to say that because people may see the private sector as the natural alternative to the public sector, an experience of petty public sector corruption could encourage individuals to trust businesses more. Alternatively, perhaps one would become more cynical about the legal protections available to them in the event of private sector malfeasance and consequently be less likely to trust the private sector. A mechanism similar to this is uncovered by Raiser et al (2008) who show that trust between firms in transition economies is higher where there is confidence in the rule of law. Using data from the Afrobarometer surveys, we find that there is a negative association between experiencing corruption and trust in the private sector. This result is robust to the inclusion of perceptions of political and business corruption and it is evident with regards to trust in all layers of the private sector.

Trust is essential when complete or legally binding contracts are impossible; in other words, in a situation of asymmetric or imperfect information (Williamson, 1985). This applies to many different settings, from customer credit and wage agreements to policy-making and is perhaps particularly important in developing economies where legal protection is weak and or costly. In addition to its role in reducing transaction costs, trust in management is positively associated with valued work behaviours and attitudes (Dirks and Ferrin, 2002). Similarly, 
variation in social capital (a concept closely related to generalized trust) affects confidence in national institutions (Brehm and Rahn, 1997). Generalized trust between citizens also facilitates resolution of collective action problems (Coleman, 1990; Putman, 1993). Relatedly, Den Butter and Mosch (2003) argue that mutual trust between government, unions and employers, the central bank, and advisory bodies was the driving force behind the Dutch recovery that began in the 1980s. La Porta et al (1997) demonstrate that trust is important for a wide range of macro level outcomes including governance and infrastructure. Trust has also been shown to matter for investment behaviour (Guiso et al, 2008; Georgarakos and Pasini, 2011; Klein and Shtudiner, 2015), labour market participation (Tu and Bulte, 2010), the level of TFP and its growth rate (Bjørnskov and Méon, 2015), and human and physical capital accumulation (Dearmon and Grier, 2011). Interpersonal trust is also important in terms of support for and the effectiveness of redistributive policies (Daniele and Geys, 2015; Bergh and Bjørnskov, 2014). Another strand of the trust literature has shown that generalised trust facilitates deregulation of business (Heinemann and Tanz, 2008; Aghion at al 2010; Pinotti, 2012; Leibrecht and Pitlik, 2015) which is a factor that has been shown to matter in terms of growth and development (Djankov et al, 2006; Gillanders and Whelan, 2014). Although Peiró-Palomino and Tortosa-Ausina (2013) demonstrate that the beneficial effect of trust on GDP per capita varies with the level of development, the literature thus suggests that, on average, a high level of trust has the potential to make a society better off and more equal.

While trust tends to lower transactions costs, corruption can act as an unofficial tax on many activities and can also increase uncertainty. For example, corruption increases the burden of regulation and red tape (Guriev, 2004; Breen and Gillanders, 2012). Huntington (1968) argues that corruption could "grease the wheels" of economic activity in poor institutional settings and both Méon and Weill (2010) and Dreher and Gassebner (2011) provide some evidence in support of this assertion. However, the weight of evidence suggests that corruption is damaging in terms of many outcomes at the individual, firm, and country level.

The importance of trust for economic outcomes has given rise to a literature that has sought to understand the causes and correlates of trust. These papers tend to study mainly generalized trust, i.e. trust towards other people (Glaeser et al, 1999; Alesina and Ferrara, 2002; Bjornskov, 2007; Dohmen et al, 2008; Yamamura, 2008; Corbacho et al, 2015). Given the apparent importance of trust and corruption to an economy, it should come as no surprise that the links between corruption and dimensions of trust have been studied in the past. Many authors have concluded that the perception of corruption and/or an experience of corruption 
are highly corrosive to trust in the state and its agents (Seligson, 2002; Anderson and Tverdova, 2003; Chang and Chu, 2006; Armah-Attoh et al, 2007; Lavallee et al, 2008; Morris and Klesner, 2010; Blanco, 2013), to interpersonal trust (Seligson, 2002; Uslaner, 2004; Banerjee, 2015), and even to opinions regarding non-domestic institutions (Torgler, 2008; Breen and Gillanders, 2015).

Our paper, however, is primarily concerned with explaining trust in the broad institutions of the private sector. One can readily see how this type of trust is vital for the efficient functioning of an economy. Individuals interact with the private sector as consumers, workers, investors, and even as policy-makers and regulators. If they have little trust in the private sector then they will make different choices than those who trust the private sector to deliver on its promises regarding the quality of goods, wage contracts, credit contracts, or regulation. This aspect of trust has received some attention in the recent past. Adams et al. (2010) study the determinants of distrust in corporations and show that ideological and psychological traits such as liberalism and cynicism correlate with corporate distrust. Ingenhoff and Sommer (2010) study trust in companies and CEOs and conclude that trust in companies and trust in CEOs differ and are influenced by different factors.

The remainder of this paper proceeds as follows. Section 2 describes our data and empirical approach. Section 3 presents our results and Section 4 concludes.

\section{DATA AND APPROACH}

For the most part, the data for this paper comes from Round 2 of the Afrobarometer. The Afrobarometer project collects data from representative surveys and contains information on attitudes and social and economic conditions. ${ }^{1}$ Round 2 was carried out in 2002-2003 and covered Botswana, Cape Verde, Ghana, Kenya, Lesotho, Malawi, Mali, Mozambique, Namibia, Nigeria, Senegal, South Africa, Tanzania, Uganda, Zambia, and Zimbabwe. While many rounds have information on corruption experience, only Round 2 has information regarding trust in private sector institutions.

\footnotetext{
${ }^{1}$ The data, the original questionnaire, and the full methodology can be obtained from www.afrobarometer.org.
} 
We have measures of trust in the private sector at three levels: big private corporations, small business and shopkeepers, and traders in local markets. They come from the following survey question:

How much do you trust each of the following, or haven't you heard enough about them to say?

- Big private corporations

- Small businesses/shopkeepers

- Traders in local markets

Respondents could answer on a scale from "not at all” to "a very great deal.” While we use the original ordered responses as a robustness test, for simplicity in most of the analysis below we use a dummy variable for each level of the private sector that takes a value of zero if the answer was "Not at all” or "A little bit" and a value of one if the answer was "A lot" or “A very great deal”. Note that we have information regarding people's attitudes to the entire spectrum of private sector activity; from the large and opaque corporations to the familiar and knowable local traders. We examine each of these separately as these issues of transparency and familiarity might give rise to qualitatively or quantitatively different relationships between public sector corruption and trust in the private sector depending on the level in question.

Our chief explanatory variable of interest captures an individual's experiences of petty corruption. We make use of the answers to the following questions:

In the past year, how often (if ever) have you had to pay a bribe, give a gift, or do a favour to government officials in order to:

- Get a document or a permit?

- Get a child into school?

- Get a household service (like piped water, electricity, or phone)?

- Avoid a problem with the police (like passing a checkpoint or avoiding a fine or arrest)?

Each of these questions can be answered "never”, “once or twice”, "a few times” and "often” to which we assign the values from 0 to 3 respectively. By summing over these four categories, we obtain an index of bribery experience that ranges from 0 to 12 , where higher 
values correspond to a more extensive experience of paying bribes. ${ }^{2}$ Note that the question explicitly refers to bribes paid to government officials. Such self-reported information could in principle be subject to biases and lead to an endogeneity problem in that less trusting people might be prone to embellishing their corruption experience history. Thus, as a robustness test we use a simple dummy variable that takes a value of one if the respondent has any reported history at all of paying a bribe in any of the above situations. The idea here is that while people might exaggerate, they are less likely to completely invent a history of bribe paying when none exists.

Table A1 presents summary statistics and full definitions for the other variables used in the paper. One can see that trust in the private sector is middling with each of our trust dummies having a mean of around 0.4. While the means are similar, Table A2 shows that the correlations between the three types of trust (measured on the ordered 0-3 scale) are not overly high. One can hold different levels of trust for different types of actor. Looking at the correlations of these measures with another dimension of trust, trust in the courts, further supports this idea. One's trust in the private sector and one's trust in the state can be different. Table A1 also tells us that $23 \%$ of people in our sample have had some experience of bribery (in the situations that the survey asks about) in the recent past.

We estimate probit models where the private sector trust dummies are the dependent variable and corruption experience is our main explanatory variable of interest. We include country dummies to account for cross country differences in the level of trust and we cluster the standard errors at the country and region level. We control for a number of characteristics that are common in the trust literature. These are defined in Table A1. ${ }^{3}$ Our most important control is the individual's perceptions of political corruption. This is constructed from the following survey element:

How many of the following people do you think are involved in corruption, or haven't you heard enough about them to say?

- $\quad$ The President and Officials in his Office

- Elected leaders, such as parliamentarians or local councillors

- Government officials

\footnotetext{
${ }^{2}$ Round 2 also asks about bribes paid to cross a border and "for anything else." We omit these as in the former case we do not know if the bribe was paid to agents of the respondent's own country or of another and in the latter case very few people indicated that they had done so. This also allows us to generate the exact same index when we use the Round 3 data to study interpersonal trust.

${ }^{3}$ Our results are robust to using income decile as an alternative to the poverty index.
} 
Each of the questions can be answered "none”, "some of them”, "most of them" and "all” to which we assign the values from 0 to 3 respectively. By summing these we obtain an index of perceptions of political corruption that ranges from 0 to 9 .

We control for perceptions of corruption for several reasons. First of all, it has been well established in the literature referenced above that perceptions of political corruption are damaging to some dimensions of trust. One's experiences of petty corruption could inform one's perceptions of grand corruption and so for the purposes of our research question it is important to see if experiences play a role in private sector trust formation holding perceptions constant. In addition, Round 2 of the Afrobarometer lacks a measure of interpersonal trust. We wish to take account of an individual's general level of trust and controlling for their perceptions of political corruption is a reasonable proxy for this to the extent that perceptions of corruption reflect an expectation that some people in the society will behave in an opportunistic manner to benefit themselves at the expense of others. As an alternative we use a dummy variable capturing trust in the courts. This gives us approximately 3000 more observations.

The literature outlined in Section 1 has noted that interpersonal trust and political trust are likely endogenous with regards to corruption. The level of interpersonal trust and political trust in a society might make corruption more or less permissible, effective, and necessary, and it may also change the perceptions of corruption. While the experimental work of Banerjee (2015) concludes that corruption has an effect on trust but not the other way around, we acknowledge that this is far from a settled issue. Moreover, our finding of a negative association between the experience of corruption and private sector trust could in principle be driven by people with low trust in the private sector availing of the public sector more often and thus encountering bribery situations more often. We offer several rebuttals to these and related endogeneity concerns. Firstly, some of the situations for which we have information on bribery demands are not situations that are alternatives to the private sector. Secondly and relatedly, while this story is plausible with regards to big private corporations which can provide goods and services that have a public sector counterpart it is less convincing when it comes to small local traders. As we will see, our results are qualitatively the same at each level of the private sector and the sizes of the coefficients are also very similar. Thirdly, in some specifications we also control for perceptions of corruption in business which could shape both trust in the private sector and corruption experiences, and in a robustness check 
we control for ideology. Finally, in a robustness exercise we swap our individual experience of corruption variable for the average in the respondent's survey region. In all cases, our main result survives.

\section{RESULTS}

\subsection{Main Results}

Tables 1, 2, and 3 present our main results. Table 1 uses trust in big private corporations as the dependent variable. Column 1 tells us that those who experience bribery in the public arena are less likely to trust big private corporations. The magnitude of the marginal effect is meaningful. Someone with a bribery index score of 3 is 3.3\% less likely to express trust in private corporations. This is comparable to the effect of being a woman and to the effect of having some secondary education. Column 2 shows that perceptions of political corruption are a strong predictor of this type of trust. Column 3 presents our central finding. Even when one controls for perceptions of corruption experiencing bribery is a significant predictor of trust in the private sector.

Column 4 swaps the bribery index for the bribery dummy and reaches the same conclusion. Holding corruption perceptions constant, any experience of bribery reduces one's trust in big private corporations by $3.6 \%$. Column 5 substitutes trust in the courts for perceptions of corruption. This does not change our results. ${ }^{4}$ Finally, Column 6 allows perceptions of how corrupt foreign and local businessmen are. Neither of these variables is significant in the case of trust in big private corporations. ${ }^{5}$

\footnotetext{
${ }^{4}$ As alternatives, we also used dummies for trust in the parliament, the army and the police. Our results were unchanged.

${ }^{5}$ If we try and explain perceptions of how corrupt local and foreign businessmen are then experience of bribery matters but only if we exclude perceptions of political corruption.
} 
TABLE 1: Trust in Big Private Corporations

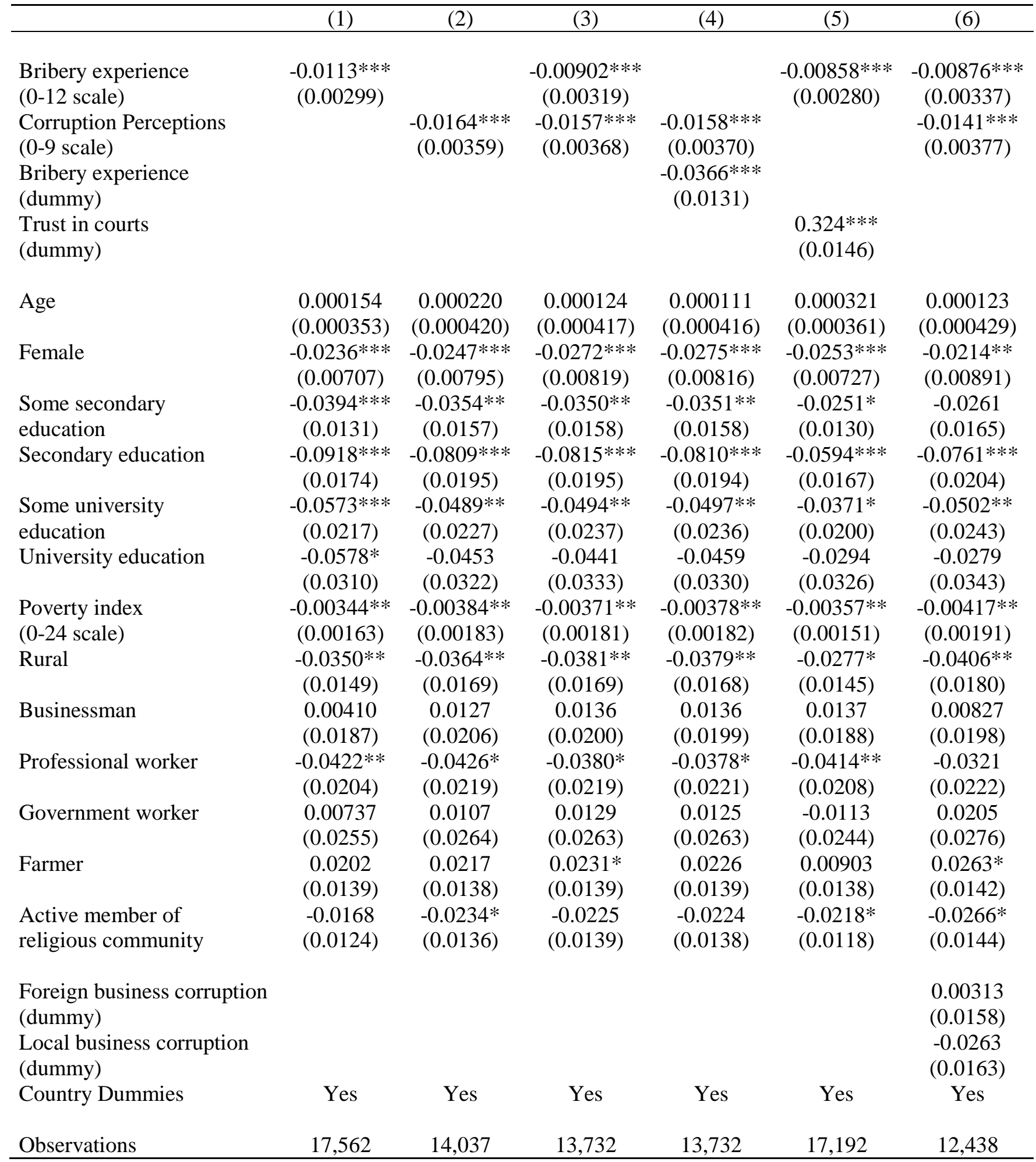
Notes: Probit marginal effects reported. The corresponding standard errors are clustered by country and region and reported in parentheses.*, **, and *** indicates significance at the $10 \%, 5 \%$ and $1 \%$ levels respectively. 
Tables 2 and 3 repeat the analysis for the other levels of the private sector. For both trust in small business and trust in local traders we mostly find the same results as we did in the case of trust in big corporations. Experiencing corruption is detrimental in terms of one's trust in these aspects of the private sector. The chief difference across the tables is the role of perceptions of how involved foreign and local businesses are in corruption. We already noted that these variables are not significant predictors of trust in large corporations. However, as Column 6 of Table 2 shows, in the case of small businesses they do seem to matter. A sense that foreign business people are involved in corruption tends to lead to more trust in small business whereas the opposite is true for the perception that local business people are involved in corruption. The significance level of corruption experiences drops to $10 \%$ in this case. Column 6 of Table 3 tells us that a perception of local businesses being involved in corruption matters for trust in local traders. Here, the perception of foreign involvement is insignificant. Corruption experiences are significant at 5\% in this specification.

Our results thus tell a clear story: petty corruption in the public sector arena has a meaningful and undesirable spill over in terms of trust in, and hence the efficient functioning of, the private sector. As mentioned in Section 1 one could think of reasons for the spill over to be negative or positive. In so far as trust is important to economic efficiency, this is a price that is paid from large corporations down to small traders in local markets, and indeed by those who interact with them and the economy in general. Perhaps surprisingly given the differences between an individual's relationship to big private corporations and to his local traders, the extent of this spill-over is much the same at each level of the private sector. Thus this exercise has uncovered a new cost to petty corruption. Corruption throws sand in the economic grease that is trust in the private sector. 
TABLE 2: Trust in Small Businesses

\begin{tabular}{|c|c|c|c|c|c|c|}
\hline & $(1)$ & $(2)$ & (3) & (4) & (5) & $(6)$ \\
\hline $\begin{array}{l}\text { Bribery experience } \\
\text { (0-12 scale) }\end{array}$ & $\begin{array}{c}-0.00924^{* * *} \\
(0.00301)\end{array}$ & & $\begin{array}{c}-0.00638^{* *} \\
(0.00311)\end{array}$ & & $\begin{array}{l}-0.00658 * * \\
(0.00301)\end{array}$ & $\begin{array}{l}-0.00668 * \\
(0.00353)\end{array}$ \\
\hline $\begin{array}{l}\text { Corruption Perceptions } \\
\text { (0-9 scale) }\end{array}$ & & $\begin{array}{l}-0.0140 * * * \\
(0.00306)\end{array}$ & $\begin{array}{l}-0.0137 * * * \\
(0.00319)\end{array}$ & $\begin{array}{l}-0.0137 * * * \\
(0.00320)\end{array}$ & & $\begin{array}{c}-0.0129 * * * \\
(0.00328)\end{array}$ \\
\hline $\begin{array}{l}\text { Bribery experience } \\
\text { (dummy) }\end{array}$ & & & & $\begin{array}{c}-0.0296^{* *} \\
(0.0121)\end{array}$ & & \\
\hline $\begin{array}{l}\text { Trust in courts } \\
\text { (dummy) }\end{array}$ & & & & & $\begin{array}{l}0.303 * * * \\
(0.0119)\end{array}$ & \\
\hline Age & $\begin{array}{l}-0.000129 \\
(0.000308)\end{array}$ & $\begin{array}{c}0.000018 \\
(0.000324)\end{array}$ & $\begin{array}{c}0.000011 \\
(0.000327)\end{array}$ & $\begin{array}{l}-0.000004 \\
(0.000325)\end{array}$ & $\begin{array}{l}-0.000012 \\
(0.000332)\end{array}$ & $\begin{array}{c}0.000156 \\
(0.000332)\end{array}$ \\
\hline Female & $\begin{array}{c}-0.0227 * * * \\
(0.00703)\end{array}$ & $\begin{array}{c}-0.0277 * * * \\
(0.00775)\end{array}$ & $\begin{array}{c}-0.0293 * * * \\
(0.00783)\end{array}$ & $\begin{array}{c}-0.0298 * * * \\
(0.00776)\end{array}$ & $\begin{array}{l}-0.0245^{* * * *} \\
(0.00676)\end{array}$ & $\begin{array}{c}-0.0225^{* * *} \\
(0.00848)\end{array}$ \\
\hline $\begin{array}{l}\text { Some secondary } \\
\text { education }\end{array}$ & $\begin{array}{c}-0.0510 * * * \\
(0.0121)\end{array}$ & $\begin{array}{c}-0.0411 * * * \\
(0.0136)\end{array}$ & $\begin{array}{c}-0.0401 * * * \\
(0.0136)\end{array}$ & $\begin{array}{c}-0.0400 * * * \\
(0.0137)\end{array}$ & $\begin{array}{c}-0.0380 * * * \\
(0.0117)\end{array}$ & $\begin{array}{l}-0.0350 * * \\
(0.0144)\end{array}$ \\
\hline Secondary education & $\begin{array}{c}-0.113 * * * \\
(0.0130)\end{array}$ & $\begin{array}{c}-0.102 * * * \\
(0.0146)\end{array}$ & $\begin{array}{c}-0.0995 * * * \\
(0.0146)\end{array}$ & $\begin{array}{c}-0.0989 * * * \\
(0.0147)\end{array}$ & $\begin{array}{l}-0.0852 * * * \\
(0.0122)\end{array}$ & $\begin{array}{c}-0.0951^{* * *} \\
(0.0161)\end{array}$ \\
\hline $\begin{array}{l}\text { Some university } \\
\text { education }\end{array}$ & $\begin{array}{c}-0.0833 * * * \\
(0.0184)\end{array}$ & $\begin{array}{c}-0.0719 * * * \\
(0.0199)\end{array}$ & $\begin{array}{c}-0.0691 * * * \\
(0.0207)\end{array}$ & $\begin{array}{c}-0.0691 * * * \\
(0.0207)\end{array}$ & $\begin{array}{c}-0.0654 * * * \\
(0.0170)\end{array}$ & $\begin{array}{c}-0.0636 * * * \\
(0.0221)\end{array}$ \\
\hline University education & $\begin{array}{c}-0.0657 * * \\
(0.0321)\end{array}$ & $\begin{array}{l}-0.0585^{*} \\
(0.0351)\end{array}$ & $\begin{array}{l}-0.0497 \\
(0.0354)\end{array}$ & $\begin{array}{l}-0.0509 \\
(0.0354)\end{array}$ & $\begin{array}{l}-0.0411 \\
(0.0327)\end{array}$ & $\begin{array}{l}-0.0310 \\
(0.0361)\end{array}$ \\
\hline $\begin{array}{l}\text { Poverty index } \\
\text { (0-24 scale) }\end{array}$ & $\begin{array}{l}-0.00274 * \\
(0.00150)\end{array}$ & $\begin{array}{l}-0.00244 \\
(0.00173)\end{array}$ & $\begin{array}{l}-0.00217 \\
(0.00167)\end{array}$ & $\begin{array}{l}-0.00220 \\
(0.00168)\end{array}$ & $\begin{array}{l}-0.00249 * \\
(0.00139)\end{array}$ & $\begin{array}{l}-0.00220 \\
(0.00176)\end{array}$ \\
\hline Rural & $\begin{array}{c}-0.0405^{* * * *} \\
(0.0143)\end{array}$ & $\begin{array}{l}-0.0404^{* *} \\
(0.0167)\end{array}$ & $\begin{array}{l}-0.0416^{* *} \\
(0.0162)\end{array}$ & $\begin{array}{l}-0.0414^{* *} \\
(0.0162)\end{array}$ & $\begin{array}{c}-0.0314^{* *} \\
(0.0142)\end{array}$ & $\begin{array}{c}-0.0385^{* *} \\
(0.0167)\end{array}$ \\
\hline Businessman & $\begin{array}{l}0.0412^{*} \\
(0.0242)\end{array}$ & $\begin{array}{c}0.0347 \\
(0.0255)\end{array}$ & $\begin{array}{c}0.0403 \\
(0.0256)\end{array}$ & $\begin{array}{c}0.0407 \\
(0.0256)\end{array}$ & $\begin{array}{l}0.0559 * * \\
(0.0234)\end{array}$ & $\begin{array}{c}0.0322 \\
(0.0253)\end{array}$ \\
\hline Professional worker & $\begin{array}{l}-0.0136 \\
(0.0211)\end{array}$ & $\begin{array}{l}-0.0114 \\
(0.0231)\end{array}$ & $\begin{array}{l}-0.00874 \\
(0.0233)\end{array}$ & $\begin{array}{l}-0.00824 \\
(0.0235)\end{array}$ & $\begin{array}{l}-0.0105 \\
(0.0213)\end{array}$ & $\begin{array}{l}-0.0132 \\
(0.0218)\end{array}$ \\
\hline Government worker & $\begin{array}{c}0.0114 \\
(0.0232)\end{array}$ & $\begin{array}{c}0.0191 \\
(0.0254)\end{array}$ & $\begin{array}{c}0.0185 \\
(0.0255)\end{array}$ & $\begin{array}{c}0.0181 \\
(0.0254)\end{array}$ & $\begin{array}{l}-0.00697 \\
(0.0228)\end{array}$ & $\begin{array}{c}0.0205 \\
(0.0259)\end{array}$ \\
\hline Farmer & $\begin{array}{c}0.0168 \\
(0.0127)\end{array}$ & $\begin{array}{l}0.0218^{*} \\
(0.0130)\end{array}$ & $\begin{array}{l}0.0226^{*} \\
(0.0133)\end{array}$ & $\begin{array}{l}0.0223^{*} \\
(0.0133)\end{array}$ & $\begin{array}{l}0.00686 \\
(0.0126)\end{array}$ & $\begin{array}{l}0.0276^{*} \\
(0.0144)\end{array}$ \\
\hline $\begin{array}{l}\text { Active member of } \\
\text { religious community }\end{array}$ & $\begin{array}{l}-0.0103 \\
(0.0136)\end{array}$ & $\begin{array}{l}-0.0136 \\
(0.0149)\end{array}$ & $\begin{array}{l}-0.0130 \\
(0.0152)\end{array}$ & $\begin{array}{l}-0.0127 \\
(0.0152)\end{array}$ & $\begin{array}{l}-0.0106 \\
(0.0135)\end{array}$ & $\begin{array}{l}-0.0166 \\
(0.0141)\end{array}$ \\
\hline $\begin{array}{l}\text { Foreign business corruption } \\
\text { (dummy) }\end{array}$ & & & & & & $\begin{array}{l}0.0347 * * \\
(0.0150)\end{array}$ \\
\hline $\begin{array}{l}\text { Local business corruption } \\
\text { (dummy) }\end{array}$ & & & & & & $\begin{array}{c}-0.0492 * * * \\
(0.0135)\end{array}$ \\
\hline Country Dummies & Yes & Yes & Yes & Yes & Yes & Yes \\
\hline Observations & 19,508 & 15,265 & 14,887 & 14,887 & 18,977 & 13,178 \\
\hline
\end{tabular}

Notes: Probit marginal effects reported. The corresponding standard errors are clustered by country and region and reported in parentheses.*, **, and *** indicates significance at the $10 \%, 5 \%$ and $1 \%$ levels respectively. 
TABLE 3: Trust in Local Traders

\begin{tabular}{|c|c|c|c|c|c|c|}
\hline & (1) & (2) & (3) & (4) & (5) & (6) \\
\hline $\begin{array}{l}\text { Bribery experience } \\
(0-12 \text { scale })\end{array}$ & $\begin{array}{c}-0.00973 * * * \\
(0.00272)\end{array}$ & & $\begin{array}{c}-0.00752 * * * \\
(0.00287)\end{array}$ & & $\begin{array}{c}-0.00690 * * * \\
(0.00262)\end{array}$ & $\begin{array}{c}-0.00787 * * \\
(0.00328)\end{array}$ \\
\hline $\begin{array}{l}\text { Corruption Perceptions } \\
\text { (0-9 scale) }\end{array}$ & & $\begin{array}{c}-0.0112 * * * \\
(0.00331)\end{array}$ & $\begin{array}{c}-0.0109 * * * \\
(0.00348)\end{array}$ & $\begin{array}{c}-0.0110 * * * \\
(0.00350)\end{array}$ & & $\begin{array}{c}-0.00857 * * \\
(0.00360)\end{array}$ \\
\hline $\begin{array}{l}\text { Bribery experience } \\
\text { (dummy) }\end{array}$ & & & & $\begin{array}{c}-0.0295^{* *} \\
(0.0121)\end{array}$ & & \\
\hline $\begin{array}{l}\text { Trust in courts } \\
\text { (dummy) }\end{array}$ & & & & & $\begin{array}{l}0.292 * * * \\
(0.0125)\end{array}$ & \\
\hline Age & $\begin{array}{l}-0.000092 \\
(0.000315)\end{array}$ & $\begin{array}{l}-0.000033 \\
(0.000363)\end{array}$ & $\begin{array}{l}-0.000070 \\
(0.000356)\end{array}$ & $\begin{array}{l}-0.000085 \\
(0.000356)\end{array}$ & $\begin{array}{c}0.000032 \\
(0.000330)\end{array}$ & $\begin{array}{c}0.000095 \\
(0.000374)\end{array}$ \\
\hline Female & $\begin{array}{c}-0.0253^{* * *} \\
(0.00738)\end{array}$ & $\begin{array}{c}-0.0345 * * * \\
(0.00734)\end{array}$ & $\begin{array}{l}-0.0386 * * * \\
(0.00776)\end{array}$ & $\begin{array}{c}-0.0388 * * * \\
(0.00772)\end{array}$ & $\begin{array}{c}-0.0265^{* * *} \\
(0.00713)\end{array}$ & $\begin{array}{c}-0.0317 * * * \\
(0.00849)\end{array}$ \\
\hline $\begin{array}{l}\text { Some secondary } \\
\text { education }\end{array}$ & $\begin{array}{c}-0.0539 * * * \\
(0.0124)\end{array}$ & $\begin{array}{c}-0.0447 * * * \\
(0.0147)\end{array}$ & $\begin{array}{c}-0.0455^{* * * *} \\
(0.0143)\end{array}$ & $\begin{array}{c}-0.0455 * * * \\
(0.0143)\end{array}$ & $\begin{array}{c}-0.0404 * * * \\
(0.0125)\end{array}$ & $\begin{array}{c}-0.0424 * * * \\
(0.0146)\end{array}$ \\
\hline Secondary education & $\begin{array}{c}-0.115^{* * *} \\
(0.0139)\end{array}$ & $\begin{array}{c}-0.101^{* * *} \\
(0.0162)\end{array}$ & $\begin{array}{c}-0.102^{* * *} \\
(0.0157)\end{array}$ & $\begin{array}{c}-0.101^{* * *} \\
(0.0157)\end{array}$ & $\begin{array}{c}-0.0885^{* * *} \\
(0.0128)\end{array}$ & $\begin{array}{c}-0.0971^{* * *} \\
(0.0165)\end{array}$ \\
\hline $\begin{array}{l}\text { Some university } \\
\text { education }\end{array}$ & $\begin{array}{l}-0.106 * * * \\
(0.0168)\end{array}$ & $\begin{array}{c}-0.0980 * * * \\
(0.0181)\end{array}$ & $\begin{array}{c}-0.100 * * * \\
(0.0188)\end{array}$ & $\begin{array}{c}-0.101^{* * * *} \\
(0.0187)\end{array}$ & $\begin{array}{c}-0.0902^{* * *} \\
(0.0160)\end{array}$ & $\begin{array}{c}-0.0926 * * * \\
(0.0201)\end{array}$ \\
\hline University education & $\begin{array}{c}-0.0954 * * * \\
(0.0290)\end{array}$ & $\begin{array}{c}-0.0910 * * * \\
(0.0310)\end{array}$ & $\begin{array}{c}-0.0843 * * * \\
(0.0313)\end{array}$ & $\begin{array}{c}-0.0858 * * * \\
(0.0310)\end{array}$ & $\begin{array}{c}-0.0696 * * \\
(0.0289)\end{array}$ & $\begin{array}{c}-0.0729 * * \\
(0.0306)\end{array}$ \\
\hline $\begin{array}{l}\text { Poverty index } \\
\text { (0-24 scale) }\end{array}$ & $\begin{array}{l}-0.00269 * \\
(0.00142)\end{array}$ & $\begin{array}{l}-0.00214 \\
(0.00163)\end{array}$ & $\begin{array}{l}-0.00176 \\
(0.00157)\end{array}$ & $\begin{array}{l}-0.00182 \\
(0.00159)\end{array}$ & $\begin{array}{l}-0.00241^{*} \\
(0.00132)\end{array}$ & $\begin{array}{l}-0.00181 \\
(0.00167)\end{array}$ \\
\hline Rural & $\begin{array}{c}-0.0390 * * * \\
(0.0137)\end{array}$ & $\begin{array}{c}-0.0465 * * * \\
(0.0154)\end{array}$ & $\begin{array}{c}-0.0426 * * * \\
(0.0149)\end{array}$ & $\begin{array}{c}-0.0425^{* * * *} \\
(0.0150)\end{array}$ & $\begin{array}{c}-0.0302^{* *} \\
(0.0136)\end{array}$ & $\begin{array}{c}-0.0403^{* * * *} \\
(0.0154)\end{array}$ \\
\hline Businessman & $\begin{array}{c}0.0305 \\
(0.0220)\end{array}$ & $\begin{array}{c}0.0315 \\
(0.0232)\end{array}$ & $\begin{array}{c}0.0343 \\
(0.0233)\end{array}$ & $\begin{array}{c}0.0341 \\
(0.0232)\end{array}$ & $\begin{array}{l}0.0437 * * \\
(0.0205)\end{array}$ & $\begin{array}{c}0.0263 \\
(0.0222)\end{array}$ \\
\hline Professional worker & $\begin{array}{l}-0.0256 \\
(0.0243)\end{array}$ & $\begin{array}{l}-0.00905 \\
(0.0249)\end{array}$ & $\begin{array}{l}-0.00955 \\
(0.0254)\end{array}$ & $\begin{array}{l}-0.00918 \\
(0.0255)\end{array}$ & $\begin{array}{l}-0.0246 \\
(0.0239)\end{array}$ & $\begin{array}{l}-0.00769 \\
(0.0278)\end{array}$ \\
\hline Government worker & $\begin{array}{l}-0.00351 \\
(0.0235)\end{array}$ & $\begin{array}{c}-0.000644 \\
(0.0252)\end{array}$ & $\begin{array}{c}-0.000480 \\
(0.0254)\end{array}$ & $\begin{array}{c}-0.000745 \\
(0.0255)\end{array}$ & $\begin{array}{l}-0.0174 \\
(0.0232)\end{array}$ & $\begin{array}{l}0.00833 \\
(0.0257)\end{array}$ \\
\hline Farmer & $\begin{array}{c}0.0112 \\
(0.0109)\end{array}$ & $\begin{array}{l}0.00864 \\
(0.0113)\end{array}$ & $\begin{array}{l}0.00859 \\
(0.0117)\end{array}$ & $\begin{array}{l}0.00829 \\
(0.0117)\end{array}$ & $\begin{array}{l}0.000413 \\
(0.0108)\end{array}$ & $\begin{array}{c}0.0153 \\
(0.0126)\end{array}$ \\
\hline $\begin{array}{l}\text { Active member of } \\
\text { religious community }\end{array}$ & $\begin{array}{l}-0.0115 \\
(0.0138)\end{array}$ & $\begin{array}{l}-0.0159 \\
(0.0156)\end{array}$ & $\begin{array}{l}-0.0152 \\
(0.0159)\end{array}$ & $\begin{array}{l}-0.0151 \\
(0.0159)\end{array}$ & $\begin{array}{l}-0.0134 \\
(0.0142)\end{array}$ & $\begin{array}{l}-0.0207 \\
(0.0155)\end{array}$ \\
\hline $\begin{array}{l}\text { Foreign business corruption } \\
\text { (dummy) } \\
\text { Local business corruption } \\
\text { (dummy) }\end{array}$ & & & & & & $\begin{array}{c}0.0114 \\
(0.0144) \\
-0.0377 * * * \\
(0.0130)\end{array}$ \\
\hline Country Dummies & Yes & Yes & Yes & Yes & Yes & Yes \\
\hline Observations & 19,548 & 15,280 & 14,891 & 14,891 & 18,987 & 13,184 \\
\hline
\end{tabular}

Notes: Probit marginal effects reported. The corresponding standard errors are clustered by country and region and reported in parentheses. ${ }^{*}, * *$, and ${ }^{* * *}$ indicates significance at the $10 \%, 5 \%$ and $1 \%$ levels respectively. 


\subsection{Robustness and Extensions}

Thus far we have used dummy indicators of trust. The main reasons for this is ease of interpretation and the fact that the people can have different conceptions of terms like "a little bit” and "a lot.” Table 4 uses the full range of information available in each indicator and reports the marginal effects obtained from ordered probit models.

The results are consistent with our main findings. A greater experience of public sector corruption is associated with an increase in the probability of trusting private corporations and small business "not at all" and "a little bit" and a decrease in the probability of trusting them "a lot” and "a very great deal.” For trust in local traders the pattern is slightly different but still consistent with our findings above. Experiencing corruption increases the probability of not trusting local traders at all and decreases the probability of expressing any degree of trust in them. The same patterns are evident with regards to the perception of political corruption. Our results are therefore robust in this regard.

Some papers in the literature referenced above have found that ideology can be a strong correlate of trust. In the case of trust in market institutions this could be a particularly important factor. The Afrobarometer allows us to consider some broad aspects of ideology. Table 5 includes evaluations of the government's handling of price stability and satisfaction with the reduced role the government plays in the economy. The first, third, and fifth columns of Table 5 show that these factors do predict higher trust in the private sector but our main result holds. The significance of these factors supports the notion that peoples' appraisals of the public and private spheres are interlinked. We also allow the respondent's attitudes and beliefs regarding the desirability or acceptability of protectionism, inequality, and the market economy to enter the specification. None of these factors are significant and their inclusion does not change our key result.

One might contend that our result is merely picking up the negative effect of experiencing bribery on interpersonal or generalised trust. Businesses are run by people and so if experiencing corruption lowers trust in people in general then it could lower trust in the private sector. Ideally we would like to include a measure of interpersonal trust in our models and see if experiencing bribery is a significant predictor of private sector trust once interpersonal trust is controlled for. Unfortunately, Round 2 of the Afrobarometer does not include such a variable. Round 3 does but lacks the information regarding trust in the private 
TABLE 4: Robustness: Ordered Probit Models

\begin{tabular}{|c|c|c|c|c|}
\hline \multirow[b]{2}{*}{ How much do you trust? } & \multicolumn{4}{|c|}{ Trust in Private Corporations } \\
\hline & Not at all & A little bit & A lot & A very great deal \\
\hline $\begin{array}{l}\text { Bribery experience } \\
\text { (0-12 scale) }\end{array}$ & $\begin{array}{c}0.0042 * * * \\
(0.0015)\end{array}$ & $\begin{array}{c}0.0020 * * * \\
(0.0008)\end{array}$ & $\begin{array}{c}-0.0034 * * * \\
(0.0012)\end{array}$ & $\begin{array}{c}-0.0028 * * * \\
(0.0010)\end{array}$ \\
\hline Corruption Perceptions & $0.011 * * *$ & $0.0053 * * *$ & $-0.0089 * * *$ & $-0.0074 * * *$ \\
\hline (0-9 scale) & $(0.0026)$ & $(0.0014)$ & $(0.0022)$ & $(0.0018)$ \\
\hline Controls & Yes & Yes & Yes & Yes \\
\hline Country Dummies & Yes & Yes & Yes & Yes \\
\hline \multirow[t]{2}{*}{ Observations } & 13,732 & 13,732 & 13,732 & 13,732 \\
\hline & \multicolumn{4}{|c|}{ Trust in Small Businesses } \\
\hline How much do you trust? & Not at all & A little bit & A lot & A very great deal \\
\hline $\begin{array}{l}\text { Bribery experience } \\
\text { (0-12 scale) }\end{array}$ & $\begin{array}{l}0.0036^{* * * *} \\
(0.0014)\end{array}$ & $\begin{array}{c}0.002 * * * \\
(0.0008)\end{array}$ & $\begin{array}{c}-0.0032^{* * *} \\
(0.0013)\end{array}$ & $\begin{array}{c}-0.0023 * * * \\
(0.0009)\end{array}$ \\
\hline Corruption Perceptions & $0.0089 * * *$ & $0.0048 * * *$ & $-0.0079 * * *$ & $-0.0057 * * *$ \\
\hline (0-9 scale) & $(0.0022)$ & $(0.0013)$ & $(0.0020)$ & $(0.0014)$ \\
\hline Controls & Yes & Yes & Yes & Yes \\
\hline Country Dummies & Yes & Yes & Yes & Yes \\
\hline \multirow[t]{2}{*}{ Observations } & 14,887 & 14,887 & 14,887 & 14,887 \\
\hline & \multicolumn{4}{|c|}{ Trust in Local Traders } \\
\hline How much do you trust? & Not at all & A little bit & A lot & A very great deal \\
\hline Bribery experience & $0.0047 * * *$ & $-0.0020^{* * *}$ & $-0.0039 * * *$ & $-0.0028 * * *$ \\
\hline Corruption Perceptions & $\begin{array}{l}(0.0013) \\
0.008 * * *\end{array}$ & $\begin{array}{c}(0.000 /) \\
-0.0035^{* * *}\end{array}$ & $\begin{array}{c}(0.0011) \\
-0.0067 * * *\end{array}$ & $\begin{array}{c}(0.0008) \\
-0.0048 * * *\end{array}$ \\
\hline (0-9 scale) & $(0.0023)$ & $(0.0011)$ & $(0.002)$ & $(0.0014)$ \\
\hline Controls & Yes & Yes & Yes & Yes \\
\hline Country Dummies & Yes & Yes & Yes & Yes \\
\hline Observations & 14,891 & 14,891 & 14,891 & 14,891 \\
\hline
\end{tabular}

Notes: Ordered Probit marginal effects reported. The corresponding standard errors are clustered by country and region and reported in parentheses.*, **, and *** indicates significance at the $10 \%, 5 \%$ and $1 \%$ levels respectively. Each specification includes the individual characteristics from the previous tables and country dummies. 
TABLE 5: Robustness: Attitudes and Ideology

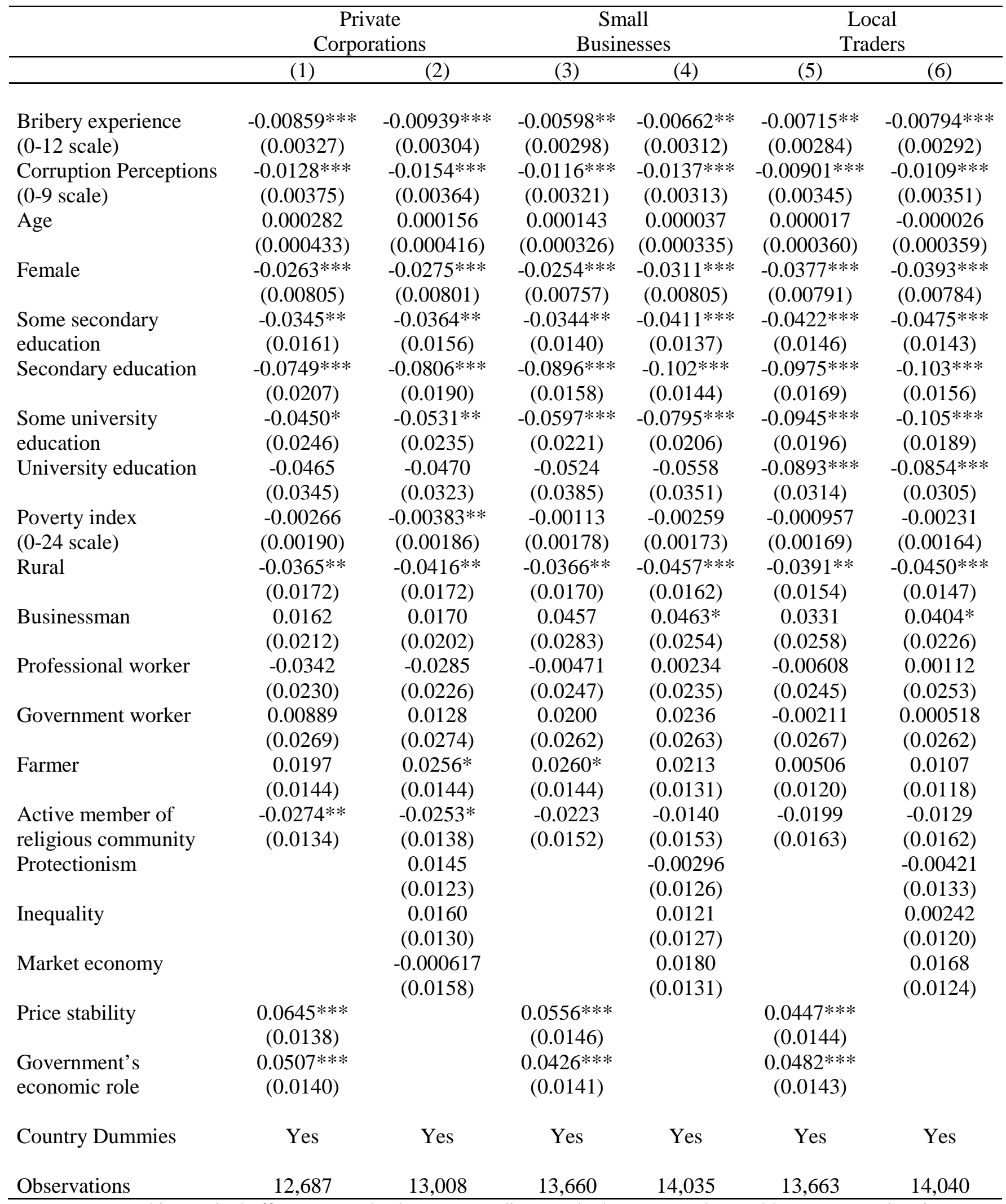
Notes: Probit marginal effects reported. The corresponding standard errors are clustered by country and region and reported in parentheses. ${ }^{*},{ }^{* *}$, and ${ }^{* * *}$ indicates significance at the $10 \%, 5 \%$ and $1 \%$ levels respectively. 
sector. We therefore take a different approach. We use the Round 3 information regarding interpersonal trust and see if bribery experience is a significant predictor of this form of trust.

From the question "Generally speaking, would you say that most people can be trusted or that you must be very careful in dealing with people?" we generate a dummy variable that takes a value of one if the answer is "Most people can be trusted" and zero if the answer is "You must be very careful." 83.26\% of the sample gave the latter answer. Barring a slight change to what is included in the poverty index the explanatory variables are the same as those used in the analysis of private sector trust using the Round 2 data.

Table 6 presents the results of this analysis. In none of our specifications is an experience of corruption statistically significantly related to interpersonal trust - even when perceptions are excluded in Column 1. This offers some defence to the charge that our result regarding private sector trust is simply reflecting an effect of corruption experiences on trust in general. While this puts us at odds with some of the findings in the existing literature, it is important to note that in common with much of the literature our results indicate that perceptions of political corruption are associated with lower interpersonal trust. As to the discrepancy between our finding and those of others' regarding the effect experiences of corruption have on interpersonal trust, we suspect that context probably matters very strongly for this relationship. In an environment where corruption is a regular occurrence in one's life it may be the case that a recent history of involvement with bribery does not alter one's opinions of people in general. 
TABLE 6: Interpersonal Trust

\begin{tabular}{|c|c|c|c|c|c|}
\hline & (1) & (2) & (3) & (4) & (5) \\
\hline $\begin{array}{l}\text { Bribery experience } \\
(0-12 \text { scale) }\end{array}$ & $\begin{array}{c}0.00056 \\
(0.00047)\end{array}$ & & $\begin{array}{c}0.0006 \\
(0.0005)\end{array}$ & & $\begin{array}{c}0.00048 \\
(0.00048)\end{array}$ \\
\hline $\begin{array}{l}\text { Corruption Perceptions } \\
\text { (0-9 scale) }\end{array}$ & & $\begin{array}{c}-0.005 * * * \\
(0.0018)\end{array}$ & $\begin{array}{c}-0.0053 * * * \\
(0.0018)\end{array}$ & $\begin{array}{c}-0.0052^{* * *} \\
(0.0018)\end{array}$ & \\
\hline $\begin{array}{l}\text { Bribery } \\
\text { experience (dummy) }\end{array}$ & & & & $\begin{array}{l}-0.0062 \\
(0.0103)\end{array}$ & \\
\hline $\begin{array}{l}\text { Trust in } \\
\text { courts (dummy) }\end{array}$ & & & & & $\begin{array}{r}0.04 * * * \\
(0.008)\end{array}$ \\
\hline Age & $\begin{array}{c}0.00049 * * * \\
(0.00018)\end{array}$ & $\begin{array}{c}0.0005^{* * *} \\
(0.0002)\end{array}$ & $\begin{array}{c}0.00053^{* * *} \\
(0.0002)\end{array}$ & $\begin{array}{l}0.0005^{* *} \\
(0.0002)\end{array}$ & $\begin{array}{c}0.00052^{* * * *} \\
(0.0002)\end{array}$ \\
\hline Female & $\begin{array}{l}-0.0011 \\
(0.0053)\end{array}$ & $\begin{array}{c}0.0036 \\
(0.0055)\end{array}$ & $\begin{array}{c}0.0039 \\
(0.0055)\end{array}$ & $\begin{array}{c}0.0037 \\
(0.0055)\end{array}$ & $\begin{array}{l}-0.0016 \\
(0.0052)\end{array}$ \\
\hline $\begin{array}{l}\text { Some } \\
\text { secondary education }\end{array}$ & $\begin{array}{c}-0.0336 * * * \\
(0.0083)\end{array}$ & $\begin{array}{l}-0.0347 * * * \\
(0.0086)\end{array}$ & $\begin{array}{c}-0.0348 * * * \\
(0.0086)\end{array}$ & $\begin{array}{c}-0.0354 * * * \\
(0.0086)\end{array}$ & $\begin{array}{l}-0.031 * * * \\
(0.008)\end{array}$ \\
\hline $\begin{array}{l}\text { Secondary } \\
\text { education }\end{array}$ & $\begin{array}{c}-0.0297 * * * \\
(0.0103)\end{array}$ & $\begin{array}{c}-0.0324 * * * \\
(0.011)\end{array}$ & $\begin{array}{c}-0.0308 * * * \\
(0.0111)\end{array}$ & $\begin{array}{c}-0.0314 * * * \\
(0.011)\end{array}$ & $\begin{array}{c}-0.025 * * \\
(0.01)\end{array}$ \\
\hline $\begin{array}{l}\text { Some } \\
\text { university education }\end{array}$ & $\begin{array}{c}-0.0553^{* * *} \\
(0.0119)\end{array}$ & $\begin{array}{c}-0.053^{* * *} \\
(0.0117)\end{array}$ & $\begin{array}{c}-0.0523 * * * \\
(0.0117)\end{array}$ & $\begin{array}{c}-0.0526 * * * \\
(0.0117)\end{array}$ & $\begin{array}{c}-0.051^{* * *} \\
(0.0119)\end{array}$ \\
\hline $\begin{array}{l}\text { University } \\
\text { education }\end{array}$ & $\begin{array}{l}-0.0132 \\
(0.0196)\end{array}$ & $\begin{array}{l}-0.0067 \\
(0.022)\end{array}$ & $\begin{array}{l}-0.0071 \\
(0.0217)\end{array}$ & $\begin{array}{l}-0.0073 \\
(0.0217)\end{array}$ & $\begin{array}{l}-0.0078 \\
(0.0196)\end{array}$ \\
\hline $\begin{array}{l}\text { Poverty index } \\
\text { (0-24 scale) }\end{array}$ & $\begin{array}{c}-0.0003 \\
(0.00078)\end{array}$ & $\begin{array}{l}-0.00004 \\
(0.00083)\end{array}$ & $\begin{array}{l}-0.00012 \\
(0.00085)\end{array}$ & $\begin{array}{l}-0.00003 \\
(0.0008)\end{array}$ & $\begin{array}{l}-0.0003 \\
(0.0008)\end{array}$ \\
\hline Rural & $\begin{array}{c}-0.0323 * * * \\
(0.0093)\end{array}$ & $\begin{array}{c}-0.0304 * * * \\
(0.0098)\end{array}$ & $\begin{array}{c}-0.0298 * * * \\
(0.0098)\end{array}$ & $\begin{array}{c}-0.029 * * * \\
(0.0099)\end{array}$ & $\begin{array}{c}-0.0313^{* * *} \\
(0.0091)\end{array}$ \\
\hline Businessman & $\begin{array}{l}-0.0078 \\
(0.0123)\end{array}$ & $\begin{array}{l}-0.0111 \\
(0.0130)\end{array}$ & $\begin{array}{l}-0.0119 \\
(0.0127)\end{array}$ & $\begin{array}{l}-0.0127 \\
(0.0128)\end{array}$ & $\begin{array}{l}-0.0038 \\
(0.0127)\end{array}$ \\
\hline $\begin{array}{l}\text { Professional } \\
\text { worker }\end{array}$ & $\begin{array}{l}-0.0164 \\
(0.0173)\end{array}$ & $\begin{array}{l}-0.0166 \\
(0.0186)\end{array}$ & $\begin{array}{l}-0.0185 \\
(0.0186)\end{array}$ & $\begin{array}{c}-0.019 \\
(0.0185)\end{array}$ & $\begin{array}{l}-0.018 \\
(0.017)\end{array}$ \\
\hline $\begin{array}{l}\text { Government } \\
\text { worker }\end{array}$ & $\begin{array}{l}-0.0298 * \\
(0.0166)\end{array}$ & $\begin{array}{c}-0.0356 * * \\
(0.0162)\end{array}$ & $\begin{array}{l}-0.035^{*} \\
(0.0165)\end{array}$ & $\begin{array}{l}-0.036 * * \\
(0.0164)\end{array}$ & $\begin{array}{c}-0.03 * \\
(0.0163)\end{array}$ \\
\hline Farmer & $\begin{array}{c}0.013 * \\
(0.0076)\end{array}$ & $\begin{array}{c}0.0136 \\
(0.0091)\end{array}$ & $\begin{array}{c}0.014 \\
(0.009)\end{array}$ & $\begin{array}{l}0.0145 \\
(0.009)\end{array}$ & $\begin{array}{c}0.013^{*} \\
(0.0079)\end{array}$ \\
\hline $\begin{array}{l}\text { Active member } \\
\text { of religious community }\end{array}$ & $\begin{array}{c}0.0034 \\
(0.0074)\end{array}$ & $\begin{array}{l}-0.0012 \\
(0.0082)\end{array}$ & $\begin{array}{l}-0.0016 \\
(0.0082)\end{array}$ & $\begin{array}{l}-0.0016 \\
(0.0082)\end{array}$ & $\begin{array}{c}0.0037 \\
(0.0075)\end{array}$ \\
\hline Country Dummies & Yes & Yes & Yes & Yes & Yes \\
\hline Observations & 22,365 & 17,335 & 17,085 & 17,085 & 21,150 \\
\hline
\end{tabular}

Notes: Probit marginal effects reported. The corresponding standard errors are clustered by country and region and reported in parentheses. ${ }^{*}{ }^{* *}$, and ${ }^{* * *}$ indicates significance at the $10 \%, 5 \%$ and $1 \%$ levels respectively. 
One environmental factor that could be of particular importance to the trust-corruption relationship is whether the individual is living in a democracy. The theoretical model of Mohtadi and Roe (2003) and the empirical analysis of Rock (2009) suggest that democracy is an important factor with regards to the prevalence of corruption and that corruption follows an inverted $U$ shape as the democracy matures. In addition to differing levels of corruption, norms and expectations regarding behaviour and punishment may be different in a context of autocratic rule and such differences could in turn influence how people react and adapt to an experience of corruption. To see if our relationships of interest vary according to regime type, we split the sample using the Polity IV measure which takes values from -10 (autocracy) to 10 (full democracy) (Marshall, 2013). A score of 6 or greater is taken to reflect a democracy. We take the average value of the Polity measure over the period 1994-2003. Table 7 shows that in the both types of regime bribery experience is negatively associated with trust in big private corporations. However, the estimated magnitude of this negative spill-over is greater in democracies. This difference in magnitude is also evident with regards to the other dimensions of private sector trust although the statistical significance of these marginal effects falls short of the $5 \%$ level for the most part. The corruption perceptions variable is always significant and interestingly the size of the association seems to be less influenced by regime type than in the case of the experience variable. We conclude from this exercise that while democracy may strengthen the relationship between experiencing corruption and private sector trust, such a relationship is evident in both broad regime types (at least with regards to big private corporations).

We noted above that our results may suffer from reverse causality arising from a tendency for people with little trust in the private sector to utilise the public sector more readily or more often. To address this, we re-ran our main models and our sample splits replacing the individual bribery experience variable with the regional (i.e. sub-national survey area) average of the bribery experience indicator. Table 8 presents the results. Columns 1, 4, and 7 use the full sample and the results support our main finding. The greater the average bribery experience in an individual's region, the less likely he or she is to trust the institutions of the private sector. This is also true when we restrict our sample to non-democratic regimes and in all cases bar trust in small business when we examine only democracies. Once again the marginal effect of bribery experience on trust in private corporations is substantially larger in democracies than in non-democratic regimes though the magnitudes are similar in each regime type for the other trust outcomes. 
TABLE 7: Sample Splits by Democracy

\begin{tabular}{lcccccc}
\hline & \multicolumn{3}{c}{ NON DEMOCRACIES } & \multicolumn{3}{c}{ DEMOCRACIES } \\
& $\begin{array}{l}\text { Private } \\
\text { Corporations }\end{array}$ & $\begin{array}{c}\text { Small } \\
\text { Businesses }\end{array}$ & $\begin{array}{c}\text { Local } \\
\text { Traders }\end{array}$ & $\begin{array}{c}\text { Private } \\
\text { Corporations }\end{array}$ & $\begin{array}{c}\text { Small } \\
\text { Businesses }\end{array}$ & $\begin{array}{c}\text { Local } \\
\text { Traders }\end{array}$ \\
\hline & $(1)$ & $(2)$ & $(3)$ & $(4)$ & $(5)$ & $(6)$ \\
\hline Bribery experience & $-0.0071^{* *}$ & -0.0047 & $-0.0068^{* *}$ & $-0.0191^{* *}$ & $-0.0141^{*}$ & $-0.012^{*}$ \\
$(0-12$ scale $)$ & $(0.00337)$ & $(0.00334)$ & $(0.00315)$ & $(0.00828)$ & $(0.00814)$ & $(0.00689)$ \\
Corruption Perceptions & $-0.0172^{* * *}$ & $-0.0147^{* * *}$ & $-0.0105^{* *}$ & $-0.0137^{* *}$ & $-0.0126^{* * *}$ & $-0.0111^{* *}$ \\
$(0-9$ scale $)$ & $(0.00473)$ & $(0.00451)$ & $(0.00487)$ & $(0.00548)$ & $(0.00413)$ & $(0.00462)$ \\
Controls & Yes & Yes & Yes & Yes & Yes & Yes \\
Country Dummies & Yes & Yes & Yes & Yes & Yes & Yes \\
Observations & 8,180 & 9,018 & 9,072 & 5,552 & 5,869 & 5,819 \\
\hline
\end{tabular}

Notes: Probit marginal effects reported. The corresponding standard errors are clustered by country and region and reported in parentheses.*, **, and *** indicates significance at the $10 \%, 5 \%$ and $1 \%$ levels respectively. Each specification includes the individual characteristics from tables 1, 2, and 3, and country dummies.

TABLE 8: Robustness: Regional Average Bribery Experience

\begin{tabular}{|c|c|c|c|c|c|c|c|c|c|}
\hline & \multicolumn{3}{|c|}{ Private Corporations } & \multicolumn{3}{|c|}{ Small Businesses } & \multicolumn{3}{|c|}{ Local Traders } \\
\hline & ALL & DEM & NON-DEM & ALL & DEM & NON-DEM & ALL & DEM & NON-DEM \\
\hline & (1) & (2) & (3) & (4) & (5) & (6) & (7) & (8) & (9) \\
\hline $\begin{array}{l}\text { Average Bribery } \\
\text { experience }\end{array}$ & $-0.0917 * * *$ & $-0.1618 * * *$ & $-0.076 * * *$ & $-0.0751 * * *$ & -0.082 & $-0.073 * * *$ & $-0.0848 * * *$ & $-0.095 * *$ & $-0.084 * * *$ \\
\hline (0-12 scale) & $(0.0214)$ & $(0.051)$ & $(0.0225)$ & $(0.0219)$ & $(0.058)$ & $(0.0231)$ & $(0.0192)$ & $(0.0458)$ & $(0.0210)$ \\
\hline $\begin{array}{l}\text { Corruption Perceptions } \\
\text { (0-9 scale) }\end{array}$ & $\begin{array}{c}-0.0156 * * * \\
(0.00355)\end{array}$ & $\begin{array}{c}-0.014 * * * \\
(0.0054)\end{array}$ & $\begin{array}{c}-0.0165 * * * \\
(0.00450)\end{array}$ & $\begin{array}{c}-0.0132 * * * \\
(0.00304)\end{array}$ & $\begin{array}{c}-0.0128 * * * \\
(0.0041)\end{array}$ & $\begin{array}{c}-0.0136 * * * \\
(0.00422)\end{array}$ & $\begin{array}{c}-0.0103 * * * \\
(0.0033)\end{array}$ & $\begin{array}{c}-0.0111^{* *} \\
(0.0045)\end{array}$ & $\begin{array}{l}-0.0094 * * \\
(0.00455)\end{array}$ \\
\hline Controls & Yes & Yes & Yes & Yes & Yes & Yes & Yes & Yes & Yes \\
\hline Country Dummies & Yes & Yes & Yes & Yes & Yes & Yes & Yes & Yes & Yes \\
\hline Observations & 14,037 & 5,603 & 8,434 & 15,265 & 5,933 & 9,332 & 15,280 & 5,886 & 9,394 \\
\hline
\end{tabular}

Notes: Probit marginal effects reported. The corresponding standard errors are clustered by country and region and reported in parentheses.*, **, and *** indicates significance at the $10 \%, 5 \%$ and $1 \%$ levels respectively. Each specification includes the individual characteristics from tables 1, 2, and 3, and country dummies 
Finally, in Table 9 we explore whether public sector corruption in certain situations might be particularly damaging in terms of private sector trust. To do this we include a dummy for each corruption situation in our models. These take a value of one if the respondent has any experience of paying a bribe in the situation in question and zero otherwise. The results of this exercise paint an interesting picture. Having to pay a bribe for a household service is significant in five out of six of our models. Only in the case of trust in small businesses where we control for perceptions of corruption is it insignificant. It is interesting that the situation which is most strongly associated with lowering trust in the private sector is also the situation that is most similar to a private sector transaction. The survey prompts people to think of services "like piped water, electricity, or phone." Services like these can be provided by private sector corporations in many countries. One might be tempted to conclude that the endogeneity problem outlined above is valid. This was the story in which low trust in private sector corporations leads individuals to engage more with the public sector and thus they are more likely to face bribe demands. It could also be the case that low trust in the private sector leads people to seek out and bribe an influential public servant to help get, for example, a phone connection. However, we must note that the effect of having to pay a bribe for services such as these on trust in local traders is also statistically significant. Even if we suspect this particular endogeneity problem is driving the result regarding trust in big private corporations, this story does not explain away the result regarding trust in local market traders. ${ }^{6}$ The only other situation where we see any evidence of a statistically meaningful effect is for trust in small businesses. Here we see some evidence that having to pay a bribe to get a document or permit lowers trust.

\section{CONCLUSIONS}

This paper has demonstrated that there is a statistically significant and meaningful relationship between sub-Saharan African survey respondents' direct experiences of public sector corruption and their trust in the private sector. Exposure to petty public sector corruption in the form of bribe paying is strongly associated with a lower probability of trusting the private sector. This relationship is evident with regards to trust in all levels of the private sector from big private corporations to small local market traders. It is robust to the inclusion of perceptions of political corruption and trust in the institutions of the state.

\footnotetext{
${ }^{6}$ If we include trust in private corporations as an explanatory variable for trust in local traders we still find a significant marginal effect of bribes for household services on trust in local traders.
} 
TABLE 9: Do Some Corruption Contexts Matter More than Others?

\begin{tabular}{|c|c|c|c|c|c|c|}
\hline & \multicolumn{2}{|c|}{$\begin{array}{c}\text { Private } \\
\text { Corporations }\end{array}$} & \multicolumn{2}{|c|}{$\begin{array}{c}\text { Small } \\
\text { Businesses }\end{array}$} & \multicolumn{2}{|c|}{$\begin{array}{c}\text { Local } \\
\text { Traders }\end{array}$} \\
\hline & (1) & (2) & (3) & (4) & (5) & (6) \\
\hline Bribe for documents & -0.0196 & -0.0151 & $-0.0287^{* *}$ & $-0.0242 *$ & -0.00942 & -0.00563 \\
\hline dumn & $(0.0136)$ & $(0.0150)$ & $(0.0145)$ & $(0.0147)$ & $(0.0135)$ & $(0.0145)$ \\
\hline Bribe for school & -0.0184 & -0.0219 & -0.0131 & -0.0116 & -0.0241 & -0.0265 \\
\hline dummy & $(0.0229)$ & $(0.0237)$ & $(0.0201)$ & $(0.0208)$ & $(0.0204)$ & $(0.0205)$ \\
\hline Bribe for service & $-0.0530 * * *$ & $-0.0435^{* *}$ & $-0.0408^{* *}$ & -0.0278 & $-0.0607 * * *$ & $-0.0567 * * *$ \\
\hline dummy & $(0.0197)$ & $(0.0207)$ & $(0.0180)$ & $(0.0187)$ & $(0.0167)$ & $(0.0181)$ \\
\hline Bribe to police & -0.00213 & 0.00753 & 0.00478 & 0.0125 & 0.00955 & 0.0239 \\
\hline dummy & $(0.0166)$ & $(0.0177)$ & $(0.0169)$ & $(0.0183)$ & $(0.0172)$ & $(0.0193)$ \\
\hline Corruption Perceptions & & $-0.0157 * * *$ & & $-0.0137 * * *$ & & $-0.0110 * * *$ \\
\hline (0-9 scale) & & $(0.00369)$ & & $(0.00319)$ & & $(0.00346)$ \\
\hline \multirow[t]{2}{*}{ Age } & 0.000149 & 0.000118 & -0.000134 & 0.000004 & -0.000093 & -0.000073 \\
\hline & $(0.000353)$ & $(0.000417)$ & $(0.000307)$ & $(0.000326)$ & $(0.000314)$ & $(0.000357)$ \\
\hline \multirow[t]{2}{*}{ Female } & $-0.0230 * * *$ & $-0.0262 * * *$ & $-0.0221 * * *$ & $-0.0286 * * *$ & $-0.0239 * * *$ & $-0.0367 * * *$ \\
\hline & $(0.00706)$ & $(0.00815)$ & $(0.00699)$ & $(0.00782)$ & $(0.00742)$ & $(0.00781)$ \\
\hline Some secondary & $-0.0394 * * *$ & $-0.0352 * *$ & $-0.0507 * * *$ & $-0.0401 * * *$ & $-0.0541 * * *$ & $-0.0459 * * *$ \\
\hline education & $(0.0130)$ & $(0.0157)$ & $(0.0121)$ & $(0.0136)$ & $(0.0123)$ & $(0.0143)$ \\
\hline \multirow[t]{2}{*}{ Secondary education } & $-0.0916^{* * *}$ & $-0.0816^{* * *}$ & $-0.112^{* * *}$ & $-0.0990 * * *$ & $-0.115^{* * *}$ & $-0.102 * * *$ \\
\hline & $(0.0172)$ & $(0.0192)$ & $(0.0129)$ & $(0.0145)$ & $(0.0138)$ & $(0.0156)$ \\
\hline Some university & $-0.0568 * * *$ & $-0.0494^{* *}$ & $-0.0824 * * *$ & $-0.0686^{* * *}$ & $-0.105^{* * *}$ & $-0.100 * * *$ \\
\hline education & $(0.0212)$ & $(0.0232)$ & $(0.0181)$ & $(0.0204)$ & $(0.0164)$ & $(0.0183)$ \\
\hline \multirow[t]{2}{*}{ University education } & $-0.0573^{*}$ & -0.0442 & $-0.0643^{* *}$ & -0.0488 & $-0.0948 * * *$ & $-0.0841 * * *$ \\
\hline & $(0.0305)$ & $(0.0327)$ & $(0.0322)$ & $(0.0354)$ & $(0.0288)$ & $(0.0311)$ \\
\hline Poverty index & $-0.00351^{* *}$ & $-0.00376^{* *}$ & $-0.00278^{*}$ & -0.00220 & $-0.00276^{*}$ & -0.00181 \\
\hline (0-24 scale) & $(0.00163)$ & $(0.00181)$ & $(0.00150)$ & $(0.00167)$ & $(0.00142)$ & $(0.00157)$ \\
\hline \multirow[t]{2}{*}{ Rural } & $-0.0340 * *$ & $-0.0372 * *$ & $-0.0398 * * *$ & $-0.0413^{* *}$ & $-0.0378 * * *$ & $-0.0413 * * *$ \\
\hline & $(0.0147)$ & $(0.0167)$ & $(0.0142)$ & $(0.0161)$ & $(0.0137)$ & $(0.0149)$ \\
\hline \multirow[t]{2}{*}{ Businessman } & 0.00404 & 0.0130 & $0.0413^{*}$ & 0.0401 & 0.0304 & 0.0335 \\
\hline & $(0.0185)$ & $(0.0198)$ & $(0.0242)$ & $(0.0255)$ & $(0.0217)$ & $(0.0230)$ \\
\hline \multirow[t]{2}{*}{ Professional worker } & $-0.0412^{* *}$ & $-0.0372 *$ & -0.0124 & -0.00790 & -0.0243 & -0.00836 \\
\hline & $(0.0204)$ & $(0.0220)$ & $(0.0212)$ & $(0.0235)$ & $(0.0244)$ & $(0.0256)$ \\
\hline \multirow[t]{2}{*}{ Government worker } & 0.00784 & 0.0137 & 0.0119 & 0.0191 & -0.00263 & 0.000970 \\
\hline & $(0.0255)$ & $(0.0263)$ & $(0.0232)$ & $(0.0253)$ & $(0.0235)$ & $(0.0254)$ \\
\hline \multirow[t]{2}{*}{ Farmer } & 0.0195 & 0.0227 & 0.0162 & $0.0221 *$ & 0.0108 & 0.00850 \\
\hline & $(0.0139)$ & $(0.0139)$ & $(0.0127)$ & $(0.0132)$ & $(0.0109)$ & $(0.0116)$ \\
\hline & -0.0163 & -0.0219 & -0.00980 & -0.0124 & -0.0111 & -0.0145 \\
\hline \multirow[t]{2}{*}{ religious community } & $(0.0124)$ & $(0.0139)$ & $(0.0135)$ & $(0.0152)$ & $(0.0138)$ & $(0.0158)$ \\
\hline & $(0.0257)$ & $(0.0371)$ & $(0.0331)$ & $(0.0314)$ & $(0.0316)$ & $(0.0392)$ \\
\hline Country Dummies & Yes & Yes & Yes & Yes & Yes & Yes \\
\hline Observations & 17,562 & 13,732 & 19,508 & 14,887 & 19,548 & 14,891 \\
\hline
\end{tabular}

Notes: Probit marginal effects reported. The corresponding standard errors are clustered by country and region and reported in parentheses. ${ }^{*}, * *$, and $* * *$ indicates significance at the $10 \%, 5 \%$ and $1 \%$ levels respectively. 
Our result also survives the inclusion of attitudinal and ideological factors that could shape one's views of private enterprise.

Given that trust in businesses is likely to be vital for the efficient functioning of an economy, this paper therefore points to a previously unknown and potentially sizable cost of petty corruption. Future and further research on these important and interesting issues could be carried out using a lab experiment. Such an approach has been taken recently by Banerjee (2015) in his study of interpersonal trust. In the current context, a well-designed experiment could offer additional, stronger, and causal evidence on the effect of public sector corruption experiences on private sector trust. In addition one could examine the effects of different types of corruption (harassment or collusive, for example). The mechanism though which corruption influences this particular form of trust could also be studied as could the potential for policies and interventions to attenuate such spill-overs.

Our central finding is that improvements in terms of citizens' exposure to petty corruption may yield a dividend in terms of trust in the private sector, and thus perhaps in terms of economic efficiency, even if their perceptions of corruption are unchanged. This is an encouraging conclusion for anti-corruption actors and adds yet another compelling argument to the case for anti-corruption efforts. 


\section{REFERENCES}

Adams, J.E., Highhouse, S. and Zickar, M.J., 2010. Understanding General Distrust of Corporations. Corporate Reputation Review, 13(1), pp. 38-51.

Afrobarometer Data, Rounds 2 and 3, available at http://www.afrobarometer.org

Aghion, P., Algan, Y., Cahuc, P., \& Shleifer, A. (2010). Regulation and Distrust. The Quarterly journal of economics, 125(3), 1015-1049.

Aidt, T., Dutta, J., \& Sena, V. (2008). Governance regimes, corruption and growth: Theory and evidence. Journal of Comparative Economics, 36(2), 195-220.

Alesina, A. and La Ferrara, E., 2002. Who trusts others? Journal of Public Economics, 85, pp. 207-234.

Anderson, C. J., \& Tverdova, Y. V. (2003). Corruption, political allegiances, and attitudes toward government in contemporary democracies. American Journal of Political Science, 47(1), 91-109.

Armah-Attoh, D., Gyimah-Boadi, E. and Chikwanha, A.B., 2007. Corruption And Institutional Trust In Africa: Implications For Democratic Development. Afrobarometer working paper No. 81. Cape Town: IDASA POS.

Banerjee, R., 2015. Corruption, Norm Violation and Decay in Social Capital. Aarhus University Economics Working Papers 2015-05. Denmark.

Bergh, A., \& Bjørnskov, C. (2014). Trust, welfare states and income equality: Sorting out the causality. European Journal of Political Economy, 35, 183-199.

Bjørnskov, C., 2007. Determinants of generalized trust: A cross-country comparison. Public Choice, 130, pp. 1-21.

Bjørnskov, C., \& Méon, P. G. (2015). The productivity of trust. World development, 70, 317331.

Blanco, L. (2013). The impact of crime and insecurity on trust in democracy and institutions. The American Economic Review, 103(3), 284-288.

Breen, M. and Gillanders, R., 2012. Corruption, Institutions and Regulation. Economics of Governance, 13(3), pp. 263-285.

Breen, M. and Gillanders, R., 2015. Political Trust, Corruption and Ratings of the IMF and the World Bank. International Interactions, 41(2), pp. 337-364.

Brehm, J. and Rahn, W., 1997. Individual level evidence for the causes and consequences of social capital. American Journal of Political Science, 41, pp. 999-1023.

Chang, E. C., \& Chu, Y. H. (2006). Corruption and trust: exceptionalism in Asian democracies?. Journal of Politics, 68(2), 259-271.

Coleman, J., 1990. Foundations of Social Theory. Cambridge, Massachusetts: Harvard University Press.

Corbacho, A., Philipp, J., \& Ruiz-Vega, M. (2015). Crime and erosion of trust: Evidence for Latin America. World Development, 70, 400-415.

Daniele, G., \& Geys, B. (2015). Interpersonal trust and welfare state support. European Journal of Political Economy, 39, 1-12. 
Dirks, K.T. and Ferrin, D.L., 2002. Trust in Leadership: Meta-Analytic Findings and Implications for Research and Practice. Journal of Applied Psychology, 87(4), pp. 611628.

Dearmon, J., \& Grier, R. (2011). Trust and the accumulation of physical and human capital. European Journal of Political Economy, 27(3), 507-519.

Den Butter, F. A., \& Mosch, R. H. (2003). The Dutch miracle: Institutions, networks, and trust. Journal of Institutional and Theoretical Economics (JITE)/Zeitschrift für die gesamte Staatswissenschaft, 362-391.

Djankov, S., McLiesh, C., \& Ramalho, R. M. (2006). Regulation and growth. Economics Letters, 92(3), 395-401.

Dohmen, T., Falk, A., Huffman, D. and Sunde, U., 2008. Representative Trust and Reciprocity: Prevalence and Determinants. Economic Inquiry, 46(1), pp. 84-90.

Donaldson, T. and Preston, L.E., 1995. The Stakeholder Theory of the Corporation: Concepts, Evidence, and Implications. Academy of Management Review, 20(1), pp. 6591.

Dreher A, Gassebner M (2011) Greasing the wheels? The impact of regulations and corruption on firm entry. Public Choice 1-20.

Fisman, R., \& Svensson, J. (2007). Are corruption and taxation really harmful to growth? Firm level evidence. Journal of Development Economics, 83(1), 63-75.

Georgarakos, D., \& Pasini, G. (2011). Trust, sociability, and stock market participation. Review of Finance, 15(4), 693-725.

Gillanders, R., \& Whelan, K. (2014). Open For Business? Institutions, Business Environment and Economic Development. Kyklos, 67(4), 535-558.

Glaeser, E.L., Laibson, D., Scheinkman, J.A. and Soutter, C.L., 1999. What is Social Capital? The Determinants of Trust and Trustworthiness. National Bureau of Economic Research Working Paper No. 7216. Cambridge: NBER.

Guiso, L., Sapienza, P., \& Zingales, L. (2008). Trusting the stock market. The Journal of Finance, 63(6), 2557-2600.

Guriev, S. (2004). Red tape and corruption. Journal of Development Economics, 73(2), 489504.

Heinemann, F., \& Tanz, B. (2008). The impact of trust on reforms. Journal of Economic Policy Reform, 11(3), 173-185.

Huntington S (1968) Political order in changing societies. Yale University Press, New Haven

Ingenhoff, D. and Sommer, K., 2010. Trust in Companies and in CEOs: A Comparative Study of the Main Influences. Journal of Business Ethics, 95, 339-355.

Klein, G. and Shtudiner, Z., 2015. Trust in others: does it affect investment decisions? Quality \& Quantity, 07/2015, pp. 1-19.

Kuenzi, M.T., 2008. Social Capital and Political Trust in West Africa. Afrobarometer working paper No. 96.

La Porta, R., Lopez-de-Silanes, F., Shleifer, A. and Vishny, R.W., 1997. Trust in Large Organizations. American Economic Review, 87, pp. 333-338. 
Lavallée, E., Razafindrakoto, M. and Roubaud, F., 2008. Corruption and Trust in Political Institutions in sub-Saharan Africa. Afrobarometer working paper No. 102.

Leibrecht, M., \& Pitlik, H. (2015). Social trust, institutional and political constraints on the executive and deregulation of markets. European Journal of Political Economy, 39, 249-268.

Marshall, M.G. (2013). Polity IV Project: Political Regime Characteristics and Transitions, 1800-2011.

Méon PG, Weill L (2010) Is corruption an efficient grease? World Dev 38(3):244-259

Mohtadi, H., \& Roe, T. L. (2003). Democracy, rent seeking, public spending and growth. Journal of Public Economics, 87(3), 445-466.

Morris, S. D., \& Klesner, J. L. (2010). Corruption and trust: Theoretical considerations and evidence from Mexico. Comparative Political Studies, 43(10), 1258-1285.

Peiró-Palomino, J., \& Tortosa-Ausina, E. (2013). Can trust effects on development be generalized? A response by quantile. European Journal of Political Economy, 32, 377390.

Pinotti, P. (2012). Trust, regulation and market failures. Review of Economics and Statistics, 94(3), 650-658.

Putnam, R., 1993. Making democracy work: civic tradition in modern Italy. Princeton: Princeton University Press.

Raiser, M., Rousso, A., Steves, F., \& Teksoz, U. (2008). Trust in transition: Cross-country and firm evidence. Journal of Law, Economics, and Organization, 24(2), 407-433.

Rock, M. T. (2009). Corruption and democracy. Journal of development studies, 45(1), 5575.

Seligson, M. A. (2002). The impact of corruption on regime legitimacy: A comparative study of four Latin American countries. The journal of Politics, 64(02), 408-433.

Torgler, Benno. 2008. "Trust in international organizations: An empirical investigation focusing on the United Nations." The Review of International Organizations 3 (1):6593.

Tu, Q., \& Bulte, E. (2010). Trust, market participation and economic outcomes: Evidence from rural China. World Development, 38(8), 1179-1190.

Williamson, O.E., 1985. The economic institutions of capitalism. New York, NY: Free Press.

Yamamura, E., 2008. Determinants of trust in a racially homogeneous society. Economics Bulletin, 26(1), pp. 1-9.

Zak, P.J. and Knack, S., 2001. Trust and Growth. The Economic Journal, 111, pp. 295-321 


\section{APPENDICES}

TABLE A1: Summary Statistics and Description of the Variables

\begin{tabular}{|c|c|c|c|}
\hline Variable & $\begin{array}{l}\text { Mean } \\
\text { (SD) }\end{array}$ & Obs & Description \\
\hline \multicolumn{4}{|c|}{ Dependent Variables } \\
\hline Trust in & 1.33 & 18990 & Level of trust in private corporations. See main text. \\
\hline Private & $(0.92)$ & & \\
\hline \multicolumn{4}{|l|}{$\begin{array}{l}\text { Corporations } \\
\text { (0-3 scale) }\end{array}$} \\
\hline Trust in Small & 1.30 & 21253 & Level of trust in small shops. See main text. \\
\hline $\begin{array}{l}\text { Businesses } \\
(0-3 \text { scale) }\end{array}$ & $(0.89)$ & & \\
\hline Trust in Local & 1.28 & 21322 & Level of trust in local traders. See main text. \\
\hline $\begin{array}{l}\text { Traders } \\
\text { (0-3 scale) }\end{array}$ & $(0.90)$ & & \\
\hline Trust in & 0.42 & 18990 & Dummy for the level of trust in private corporations. See main text. \\
\hline Private & $(0.49)$ & & \\
\hline \multicolumn{4}{|l|}{$\begin{array}{l}\text { Corporations } \\
\text { (dummy) }\end{array}$} \\
\hline Trust in Small & 0.38 & 21253 & Dummy for the level of trust in small shops. See main text. \\
\hline $\begin{array}{l}\text { Businesses } \\
\text { (dummy) }\end{array}$ & $(0.49)$ & & \\
\hline Trust in Local & 0.38 & 21322 & Dummy for the level of trust in local traders. See main text. \\
\hline $\begin{array}{l}\text { Traders } \\
\text { (dummy) }\end{array}$ & $(0.48)$ & & \\
\hline \multicolumn{4}{|c|}{ Bribery Experience } \\
\hline $\begin{array}{l}\text { Bribery } \\
\text { experience } \\
(0-12 \text { scale) }\end{array}$ & $\begin{array}{l}0.72 \\
(1.78)\end{array}$ & 23495 & Index of bribery experience. See main text. \\
\hline $\begin{array}{l}\text { Bribery } \\
\text { experience } \\
\text { (dummy) }\end{array}$ & $\begin{array}{l}0.23 \\
(0.42)\end{array}$ & 23495 & Dummy variable, equals “ 1 ” if the respondent has any bribery experience. \\
\hline $\begin{array}{l}\text { Bribe for } \\
\text { documents } \\
\text { (0-3 scale) }\end{array}$ & $\begin{array}{l}0.23 \\
(0.63)\end{array}$ & 24015 & $\begin{array}{l}\text { Bribery experience in getting document or permit, component of the bribery experience } \\
\text { index. See main text. }\end{array}$ \\
\hline $\begin{array}{l}\text { Bribe for } \\
\text { documents } \\
\text { (dummy) }\end{array}$ & $\begin{array}{l}0.14 \\
(0.35)\end{array}$ & 24015 & $\begin{array}{l}\text { Dummy variable, equals " } 1 \text { " if the respondent has bribery experience in getting } \\
\text { document or permit. }\end{array}$ \\
\hline $\begin{array}{l}\text { Bribe for } \\
\text { school } \\
\text { (0-3 scale) }\end{array}$ & $\begin{array}{l}0.14 \\
(0.51)\end{array}$ & 24108 & $\begin{array}{l}\text { Bribery experience in getting a child into school, component of the bribery experience } \\
\text { index. See main text. }\end{array}$ \\
\hline $\begin{array}{l}\text { Bribe for } \\
\text { school } \\
\text { (dummy) }\end{array}$ & $\begin{array}{l}0.09 \\
(0.29)\end{array}$ & 24108 & $\begin{array}{l}\text { Dummy variable, equals " } 1 \text { " if the respondent has bribery experience in getting a child } \\
\text { into school. }\end{array}$ \\
\hline $\begin{array}{l}\text { Bribe for } \\
\text { service } \\
\text { (0-3 scale) }\end{array}$ & $\begin{array}{l}0.145 \\
(0.53)\end{array}$ & 23748 & $\begin{array}{l}\text { Bribery experience in getting a household service, component of the bribery experience } \\
\text { index. See main text. }\end{array}$ \\
\hline $\begin{array}{l}\text { Bribe for } \\
\text { service } \\
\text { (dummy) }\end{array}$ & $\begin{array}{l}0.08 \\
(0.28)\end{array}$ & 23748 & $\begin{array}{l}\text { Dummy variable, equals " } 1 \text { " if the respondent has bribery experience in getting a } \\
\text { household service. }\end{array}$ \\
\hline \multirow{2}{*}{$\begin{array}{l}\text { Bribe to } \\
\text { police } \\
\text { ( } 0-3 \text { scale) }\end{array}$} & $\begin{array}{l}0.23 \\
(0.67)\end{array}$ & 23951 & $\begin{array}{l}\text { Bribery experience in avoiding a problem with the police, component of the bribery } \\
\text { experience index. See main text. }\end{array}$ \\
\hline & 0.12 & 23951 & Dummy variable, equals " 1 " if the respondent has bribery experience in avoiding a \\
\hline
\end{tabular}




\begin{tabular}{|c|c|c|c|}
\hline $\begin{array}{l}\text { Bribe to } \\
\text { police } \\
\text { (dummy) }\end{array}$ & $(0.33)$ & & problem with the police. \\
\hline \multicolumn{4}{|c|}{ Control Variables } \\
\hline $\begin{array}{l}\text { Corruption } \\
\text { Perceptions } \\
\text { (0-9 scale) }\end{array}$ & $\begin{array}{l}3.77 \\
(2.26)\end{array}$ & 17737 & Index of political corruption perception. See main text. \\
\hline $\begin{array}{l}\text { Corrupt } \\
\text { president } \\
\text { (0-3 scale) }\end{array}$ & $\begin{array}{l}1.12 \\
(0.90)\end{array}$ & 18750 & $\begin{array}{l}\text { Perception of the corruption in the president's office, component of the political } \\
\text { corruption perception index. See main text. }\end{array}$ \\
\hline $\begin{array}{l}\text { Corrupt } \\
\text { leaders } \\
\text { (0-3 scale) }\end{array}$ & $\begin{array}{l}1.23 \\
(0.83)\end{array}$ & 19658 & $\begin{array}{l}\text { Perception of the corruption among the elected leaders, component of the political } \\
\text { corruption perception index. See main text. }\end{array}$ \\
\hline $\begin{array}{l}\text { Corrupt } \\
\text { government } \\
\text { officials ( } 0-3 \\
\text { scale) }\end{array}$ & $\begin{array}{l}1.38 \\
(0.82)\end{array}$ & 20413 & $\begin{array}{l}\text { Perception of the corruption among government officials, component of the political } \\
\text { corruption perception index. See main text. }\end{array}$ \\
\hline Age & $\begin{array}{l}36.3 \\
(14.8)\end{array}$ & 23665 & "How old were you at your last birthday?” \\
\hline Female & $\begin{array}{l}0.50 \\
(0.50)\end{array}$ & 24301 & Equals " 1 " if the respondent is female. \\
\hline $\begin{array}{l}\text { Some } \\
\text { Secondary }\end{array}$ & $\begin{array}{l}0.36 \\
(0.48)\end{array}$ & 24229 & $\begin{array}{l}\text { "What is the highest level of education you have completed?" } \\
\text { Primary or Some Secondary Education }\end{array}$ \\
\hline $\begin{array}{l}\text { Secondary } \\
\text { Education }\end{array}$ & $\begin{array}{l}0.15 \\
(0.36)\end{array}$ & 24229 & Secondary Education \\
\hline $\begin{array}{l}\text { Some } \\
\text { University } \\
\text { Education }\end{array}$ & $\begin{array}{l}0.08 \\
(0.28)\end{array}$ & 24229 & Post-Secondary or Some University Education \\
\hline $\begin{array}{l}\text { University } \\
\text { Education }\end{array}$ & $\begin{array}{l}0.02 \\
(0.15)\end{array}$ & 24229 & University Complete or Postgraduate Education \\
\hline $\begin{array}{l}\text { Poverty index } \\
\text { (0-24 scale) }\end{array}$ & $\begin{array}{l}8.69 \\
(5.32)\end{array}$ & 23625 & $\begin{array}{l}\text { Sum of 0-4 scale indices of shortages of food, water, medical care, electricity, fuel for } \\
\text { cooking and cash income. Larger numbers indicate more poverty. See } \\
\text { www.afrobarometer.org for details of the individual questions. }\end{array}$ \\
\hline Rural & $\begin{array}{l}0.38 \\
(0.48)\end{array}$ & 24301 & Equals " 1 " if the Primary Sampling Unit is rural. \\
\hline Businessman & $\begin{array}{l}0.06 \\
(0.22)\end{array}$ & 24127 & Equals “1” if the respondent's main occupation is a businessman. \\
\hline $\begin{array}{l}\text { Professional } \\
\text { worker }\end{array}$ & $\begin{array}{l}0.03 \\
(0.18)\end{array}$ & 24127 & Equals " 1 " if the respondent's main occupation is a professional worker. \\
\hline $\begin{array}{l}\text { Government } \\
\text { worker }\end{array}$ & $\begin{array}{l}0.03 \\
(0.17)\end{array}$ & 24127 & Equals “ 1 ” if the respondent's main occupation is a government worker. \\
\hline Farmer & $\begin{array}{l}0.32 \\
(0.47)\end{array}$ & 24127 & Equals " 1 " if the respondent's main occupation is a farmer. \\
\hline $\begin{array}{l}\text { Active } \\
\text { member of } \\
\text { religious } \\
\text { community }\end{array}$ & $\begin{array}{l}0.53 \\
(0.50)\end{array}$ & 23130 & $\begin{array}{l}\text { Equals "1" if the respondent an active member of an official leader of the religious } \\
\text { group. }\end{array}$ \\
\hline $\begin{array}{l}\text { Trust in } \\
\text { Courts } \\
\text { (0-3 scale) }\end{array}$ & $\begin{array}{l}1.50 \\
(0.93)\end{array}$ & 23006 & $\begin{array}{l}\text { "How much do you trust courts, or haven't you heard enough about them to say? } \\
\text { Scale from "0"="Not at all” to 3="A very great deal". }\end{array}$ \\
\hline $\begin{array}{l}\text { Trust in } \\
\text { Courts } \\
\text { (dummy) }\end{array}$ & $\begin{array}{l}0.49 \\
(0.50)\end{array}$ & 23006 & $\begin{array}{l}\text { Dummy variable, equals " } 1 \text { " if the answer to the question above is "A lot" or "A very } \\
\text { great deal". }\end{array}$ \\
\hline
\end{tabular}




\begin{tabular}{|c|c|c|c|}
\hline $\begin{array}{l}\text { Foreign } \\
\text { business } \\
\text { corruption } \\
\text { (0-3 scale) }\end{array}$ & $\begin{array}{l}1.32 \\
(0.88)\end{array}$ & 18242 & $\begin{array}{l}\text { "How many of the foreign businessmen do you think are involved in corruption, or } \\
\text { haven’t you heard enough about them to say?" Scale from "0"="None" to " } 3 \text { "="All". }\end{array}$ \\
\hline $\begin{array}{l}\text { Foreign } \\
\text { business } \\
\text { corruption } \\
\text { (dummy) }\end{array}$ & $\begin{array}{l}0.35 \\
(0.48)\end{array}$ & 18242 & Dummy variable, equals " 1 " if the answer to the question above is " 2 " or " 3 ". \\
\hline $\begin{array}{l}\text { Local } \\
\text { business } \\
\text { corruption } \\
\text { (0-3 scale) }\end{array}$ & $\begin{array}{l}1.27 \\
(0.83)\end{array}$ & 19953 & $\begin{array}{l}\text { "How many of the local businessmen do you think are involved in corruption, or haven't } \\
\text { you heard enough about them to say?" Scale from "0"="None" to "3"="All". }\end{array}$ \\
\hline $\begin{array}{l}\text { Local } \\
\text { business } \\
\text { corruption } \\
\text { (dummy) }\end{array}$ & $\begin{array}{l}0.32 \\
(0.47)\end{array}$ & 19953 & Dummy variable, equals " 1 " if the answer to the question above is " 2 " or " 3 ". \\
\hline Protectionism & $\begin{array}{l}0.67 \\
(0.47)\end{array}$ & 22315 & $\begin{array}{l}\text { Equals " } 1 \text { " if the respondent thinks that "we must protect producers within our own } \\
\text { country by imposing tariffs that makes imported goods more expensive". }\end{array}$ \\
\hline Inequality & $\begin{array}{l}0.38 \\
(0.49)\end{array}$ & 22656 & $\begin{array}{l}\text { Equals " } 1 \text { " if the respondent thinks that "it is alright to have large differences of wealth } \\
\text { because those who work hard deserve to be rewarded". }\end{array}$ \\
\hline $\begin{array}{l}\text { Market } \\
\text { economy }\end{array}$ & $\begin{array}{l}0.47 \\
(0.50)\end{array}$ & 22753 & $\begin{array}{l}\text { Equals " } 1 \text { " if the respondent thinks that "a free market economy is preferable to a } \\
\text { government-run economy". }\end{array}$ \\
\hline Price stability & $\begin{array}{l}0.34 \\
(0.47)\end{array}$ & 23126 & $\begin{array}{l}\text { Equals "1" if the respondent thinks that current government is handling the stability of } \\
\text { prices fairly/very well. }\end{array}$ \\
\hline $\begin{array}{l}\text { Government's } \\
\text { economic role }\end{array}$ & $\begin{array}{l}0.47 \\
(0.50)\end{array}$ & 28942 & $\begin{array}{l}\text { Equals " } 1 \text { " if the respondent satisfied with the reduced role of the government in the } \\
\text { economy. }\end{array}$ \\
\hline
\end{tabular}

\section{TABLE A2: Correlation Matrix}

\begin{tabular}{|c|c|c|c|c|c|c|c|c|}
\hline & 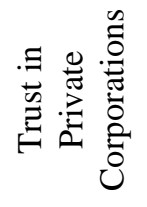 & 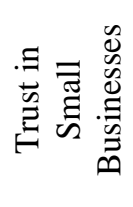 & 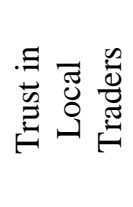 & 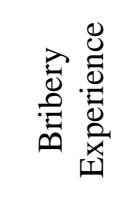 & 苛 & 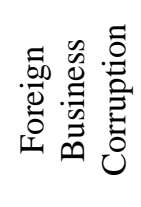 & 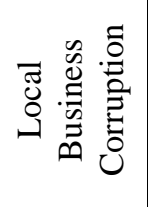 & 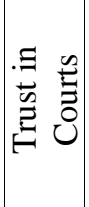 \\
\hline Trust in Private Corporations & 1 & & & & & & & \\
\hline Trust in Small Businesses & 0.6325 & 1 & & & & & & \\
\hline Trust in Local Traders & 0.5611 & 0.7727 & 1 & & & & & \\
\hline Bribery Experience & -0.0708 & -0.0446 & -0.0339 & 1 & & & & \\
\hline Political Corruption & -0.0753 & -0.0716 & -0.0555 & 0.1056 & 1 & & & \\
\hline Foreign Business Corruption & -0.0343 & -0.0396 & -0.0470 & 0.0312 & 0.3750 & 1 & & \\
\hline Local Business Corruption & -0.0379 & -0.0484 & -0.0428 & 0.0379 & 0.3805 & 0.6244 & 1 & \\
\hline Trust in Courts & 0.4025 & 0.3831 & 0.3562 & -0.0911 & -0.1549 & -0.0362 & -0.0531 & 1 \\
\hline
\end{tabular}

